\section{Case Reports in Oncology}

\title{
Metastases from Occult Breast Cancer: A Case Report of Carcinoma of Unknown Primary Syndrome
}

\author{
Erika Barbieri $^{a} \quad$ Chiara Annunziata Pasqualina Anghelone ${ }^{a}$ \\ Damiano Gentile ${ }^{a, b} \quad$ Carlotta La Raja ${ }^{a}$ b Alberto Bottini ${ }^{a}$ \\ Corrado Tinterri ${ }^{a}$ \\ ${ }^{a}$ Breast Unit, Humanitas Clinical and Research Center - IRCCS, Milan, Italy; ${ }^{b}$ Department of \\ Biomedical Sciences, Humanitas University, Milan, Italy
}

\section{Keywords}

Carcinoma of unknown primary $\cdot$ Metastatic breast cancer $\cdot$ Occult breast cancer

\begin{abstract}
Carcinoma of unknown primary (CUP) syndrome occurs when metastases from an unknown primary site spread to multiple organs. Occult breast cancer $(O B C)$ is defined as a clinically recognizable metastatic carcinoma from an undetectable primary breast tumor. It accounts for $0.3-1 \%$ of all breast cancers, often presenting with lymph node, bone, and skin metastases. Clinical and radiological examinations represent the first steps in the diagnostic algorithm for CUP syndrome from OBC. However, histological and immunohistochemical analyses, multidisciplinary team evaluation, and a multidisciplinary therapy are essential in the diagnosis and treatment of CUP syndrome from OBC. We report the case of a 52-year-old woman who underwent the removal of a parietal skin lesion. The histological and immunohistochemical analyses suggested a breast cancer origin. Clinical assessment and laboratory and radiological examinations did not locate the primary tumor. Hormone therapy was offered to the patient; however, she refused it. After 28 months, the patient reported a right cervical lump, and a total-body positron emission tomography showed dissemination of the disease to the lymph nodes and bone. A CUP syndrome from OBC was diagnosed. A multimodality approach with radiotherapy and hormone and biological therapy was started. At present, 5 years from the first presentation, the patient is asymptomatic despite the disseminated disease.
\end{abstract}




\section{Introduction}

Carcinoma of unknown primary (CUP) is defined as a biopsy-proven metastasis from a malignant disease without a detectable primary site after complete clinical and radiological examinations [1]. The "CUP syndrome" occurs when metastatic cells have spread from the unknown primary site to multiple organs, with a 2-year survival rate of $20 \%[2,3]$. This complex clinical entity accounts for approximately $2-4 \%$ of newly diagnosed cancers, commonly manifesting itself as metastases to the lymph nodes, followed by the liver, bone, and skin [3].

Occult breast cancer (OBC) was firstly described by Halsted [4] and is defined as a clinically recognizable metastatic carcinoma from an undetectable primary breast tumor. It accounts for $0.3-1 \%$ of all breast cancers, with metastasis to the axillary and cervical lymph nodes as the first presentation of a CUP syndrome [5]. The diagnostic and therapeutic algorithm for a CUP syndrome from OBC is based on its clinical presentation and on its histological and immunohistochemical analyses [6, 7]. These patients should undergo basic breast diagnostics (i.e., bilateral mammography and breast ultrasound [US]), magnetic resonance imaging (MRI) of the breast [8], and positron emission tomography (PET)/computed tomography (CT) with 2-deoxy-2-[ $\left.{ }^{18} \mathrm{~F}\right]$ fluoro-D-glucose $\left({ }^{18} \mathrm{~F}-\mathrm{FDG}\right)$ [3]. In particular, MRI of the breast allows the diagnosis of an OBC in about 75\% of women with CUP syndrome [9], and ${ }^{18} \mathrm{~F}-\mathrm{FDG}$-PET/CT identifies the primary tumor in $25-40 \%$ of these cases [10].

Histological and immunohistochemical examinations are fundamental for the diagnosis and therapy of CUP syndrome from OBC, which often requires a multidisciplinary team evaluation and a multimodality approach including surgery, radiotherapy, and hormone and systemic therapy [11]. Both mastectomy and breast preservation have been proposed for the treatment of OBC [12], with or without lymph node dissection for diagnosis and locoregional control $[13,14]$. However, a definitive consensus on the treatment of CUP syndrome from OBC has not been reached [15].

\section{Case Report}

A 52-year-old Caucasian woman with a history of celiac disease, hypertension, osteoporosis, and cigarette smoking underwent surgical removal of a skin lesion in the right parietal region which was referred as increased in size over the last 18 months. The specimen underwent two independent histological evaluations, which both agreed on the diagnosis of mucinous carcinoma localized to the derma, with positive resection margins.

The immunophenotype was: GATA3+, estrogen receptor (ER)+, progesterone receptor (PgR)+, focal positivity for GCDFP15, CK5.6+, CK19+, CK7-, CK20-, and p63-. The cellular proliferation index (MIB1) was 25-30\%. The biological profile was ER (Roche, SP1) [ASCO-CAP 2010] $>90 \%$, PgR (Roche, 1E2) [ASCO-CAP 2010] 80\%, Ki-67 index 25-30\%, and Her-2/neu (c-erbB2, Roche, 4B5) [ASCO-CAP 2013] negative. Given the intense positivity for GATA3, ER, and PgR and the focal positivity for GCDFP15, the primary hypothesis was that the lesion was a secondary location of a primary breast cancer. The case was therefore brought to the attention of our breast unit.

Clinical examination revealed the presence of a nodule in the right upper external quadrant. The nodule had the US appearance of a 6-mm cyst (BIRADS 2; Fig. 1a). A bilateral mammography revealed also the presence of a $10-\mathrm{mm}$ calcified lesion of benign appearance in the left lower quadrant (BIRADS 2; Fig. 1b). Radiological (abdominal US, MRI of the breast, and total-body ${ }^{18}$ F-FDG-PET/CT) and laboratory examinations (CA 15-3, CA 125, CA 19-9, and CEA) were within normal range and did not contribute to the localization of the OBC. 


\section{Case Reports in Oncology}

\begin{tabular}{l|l}
\hline Case Rep Oncol 2020;13:1158-1163 \\
\hline DOI: 10.1159/000510001 & $\begin{array}{l}\text { @ 2020 The Author(s). Published by S. Karger AG, Basel } \\
\text { www.karger.com/cro }\end{array}$ \\
\hline
\end{tabular}

Barbieri et al.: Carcinoma of Unknown Primary Syndrome from Occult Breast Cancer

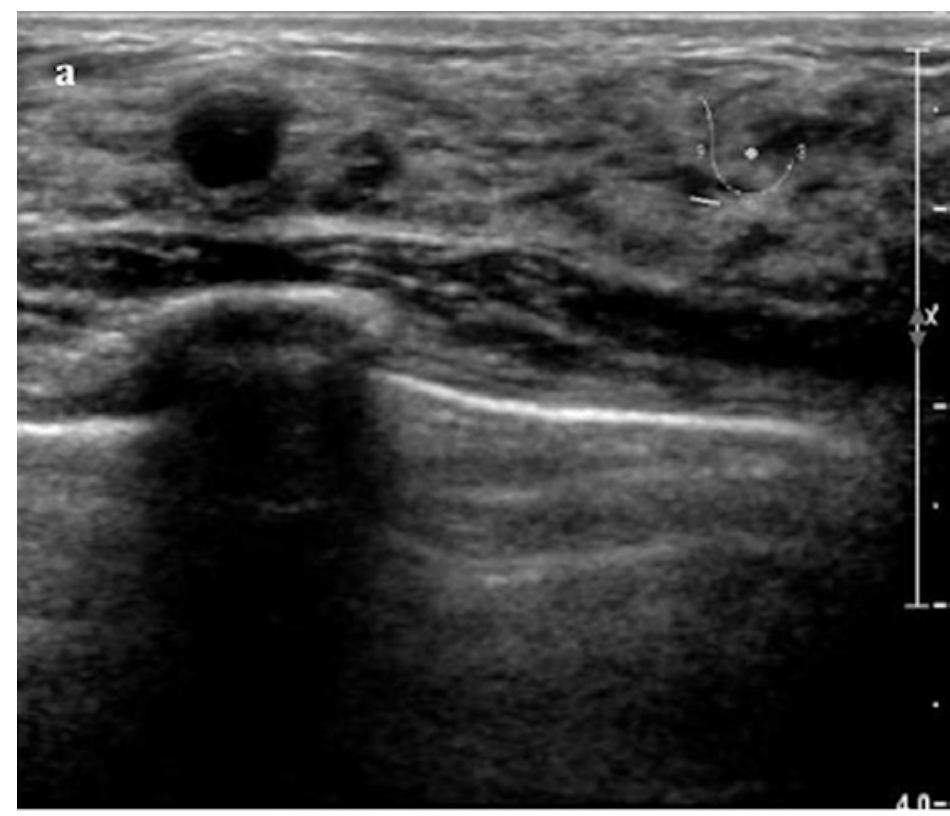

Fig. 1. a Right breast ultrasound. A 6-mm benign cyst can be observed in the upper external quadrant (BIRADS 2). b Left breast mammography. A $10-\mathrm{mm}$ calcified lesion of benign appearance can be observed in the lower quadrant (BIRADS 2).

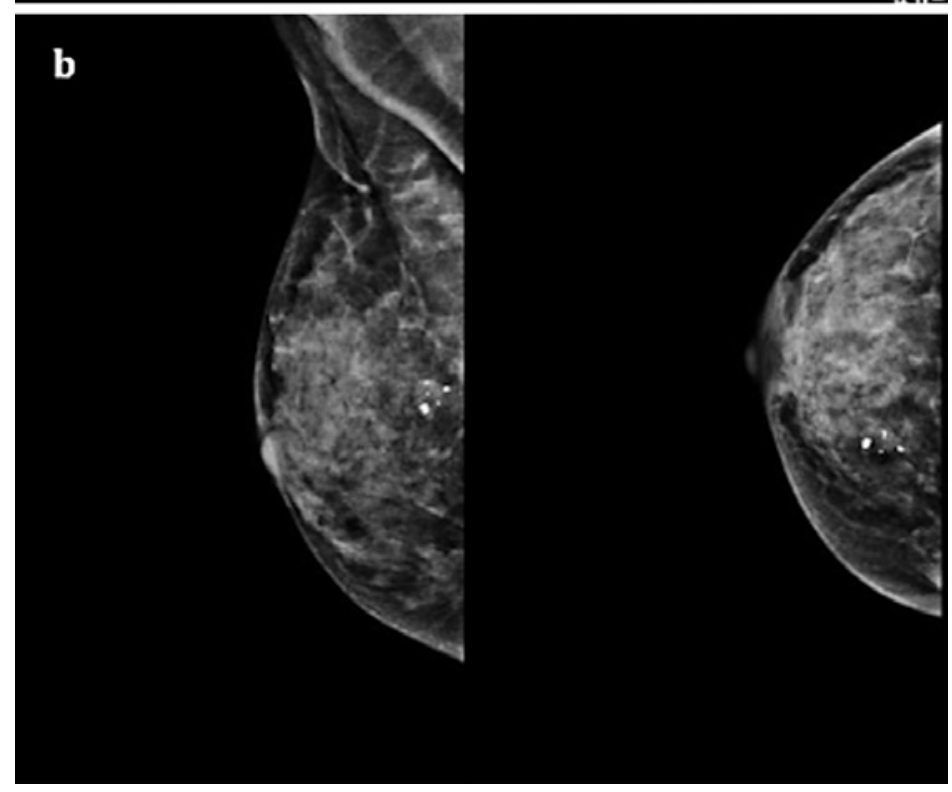

Following a breast multidisciplinary evaluation among surgeons, oncologists, radiotherapists, and pathologists, the patient underwent surgical radicalization of the right parietal skin lesion, which resulted negative for residual cancer. Therapy with tamoxifen was offered to the patient; however, she refused hormone therapy.

One year later, the patient underwent follow-up clinical assessment, bilateral mammography, and breast US, all of which resulted unchanged. The patient refused fine needle aspiration biopsy of the left lower quadrant breast nodule and agreed instead to a radiologically guided breast resection. At the histological assessment, the lesion was found to have components of typical ductal hyperplasia.

Sixteen months later, the patient noticed a right cervical lump, which underwent US evaluation, resulting in an enlarged lymph node of $28 \mathrm{~mm}$. A total-body ${ }^{18} \mathrm{~F}$-FDG-PET/CT was performed, and hypermetabolic areas were identified in the right cervical lymph nodes, vertebral column, right scapula, ribs bilaterally, sternum, pelvis, and both femurs (Fig. 2a). 


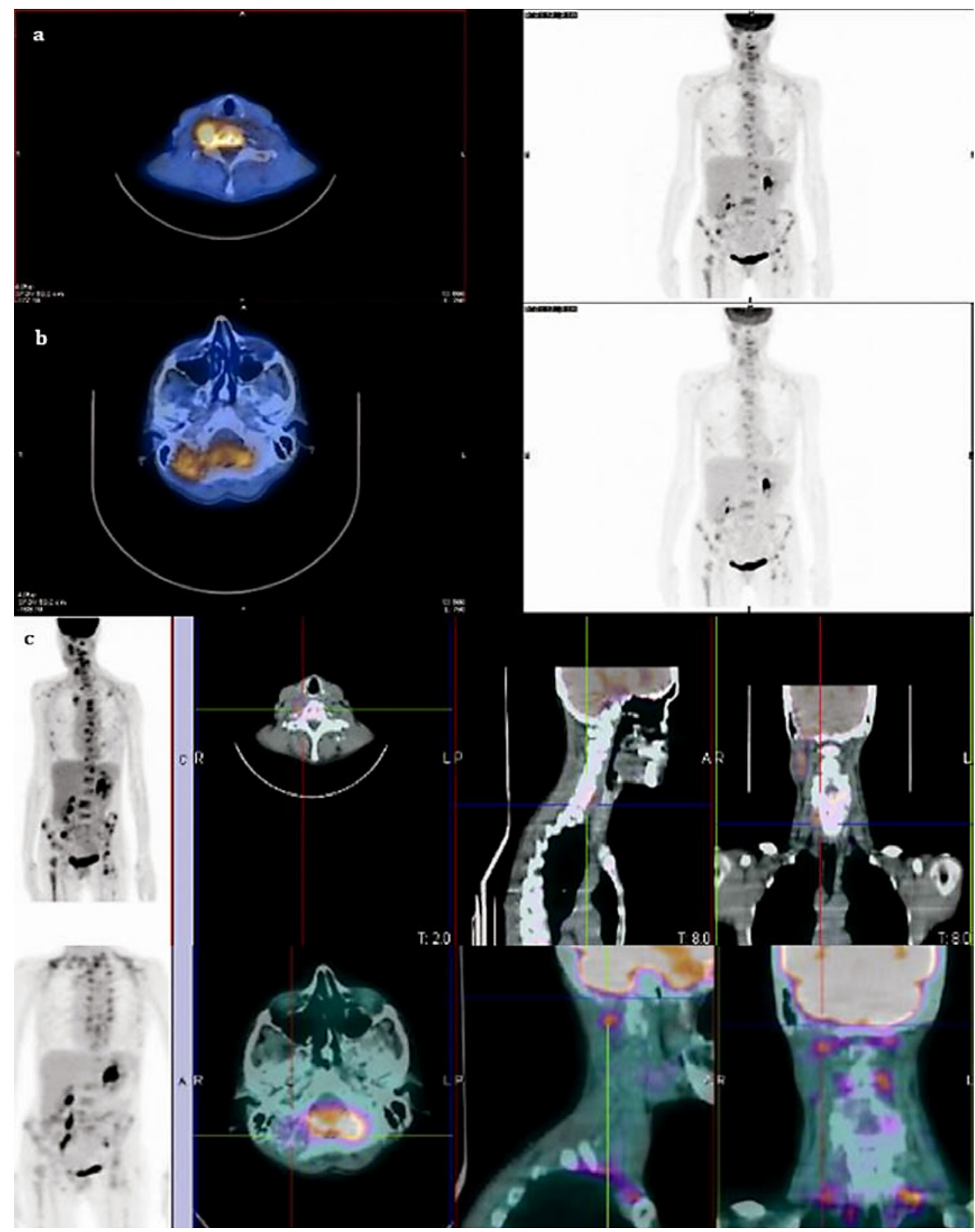

Fig. 2. a Hypermetabolic areas in the right cervical lymph nodes. b Hypermetabolic right occipital and mastoid lesions. c Slight decrease in metabolic activity in the right cervical lymph nodes, and mastoid metastasis after multimodality therapy.

Hypermetabolic right occipital and mastoid lesions were also identified (Fig. 2b). Bilateral mammography and breast US were repeated and resulted again negative. A fine needle aspiration biopsy of the enlarged right cervical lymph node was performed, being suggestive of a secondary location from breast cancer (GATA3+, ER 90\%, PgR-, Ki-67 index 20\%, and Her-2/ neu 1+). A CUP syndrome from OBC was then diagnosed. 
Therapy with letrozole, abemacilib, and weekly denosumab was started. Four months after the start of the hormone and biological therapy, the patient underwent a follow-up encephalic and spinal cord MRI, which showed a $4-\mathrm{cm}$ right mastoid lesion extending to the posterior brain fossa and compressing the right cerebellar hemisphere. Widespread vertebral lesions were confirmed. The patient did not complain of any symptoms and underwent a multimodality approach with radiotherapy ( 30 Gy in 10 sessions) and hormone and biological therapy. Ten months after the conclusion of the radiotherapy, the patient is still asymptomatic, and the last total-body ${ }^{18} \mathrm{~F}$-FDG-PET/CT showed a slight decrease in metabolic activity in the lymph nodes, bone lesions, and right mastoid metastasis (Fig. 2c).

\section{Discussion and Conclusion}

We reported a case of CUP syndrome from OBC with metastases localized to the skin, lymph nodes, and bones. The primary breast cancer remained clinically and radiologically occult; however, histological and immunohistochemical findings were fundamental to identify its origin.

There is a lack of consensus for the treatment of CUP syndrome from $\mathrm{OBC}$, and various therapeutic strategies have been proposed. After multidisciplinary team evaluation, our patient underwent a multimodality treatment including surgery, radiotherapy, and hormone and biological therapy. The prognosis for this complex oncological entity is considered very poor. However, 5 years after primary presentation, our patient is still asymptomatic despite disseminated disease.

\section{Statement of Ethics}

All procedures were conducted in accordance with the ethical standards of the Helsinki Declaration of 1975 (in its most recent version). Patient anonymity and all confidential information have been preserved. We present no identifiable material, and the patient gave informed consent for the publication of this case report. The Institutional Review Board of our hospital approved this study.

\section{Conflict of Interest Statement}

The authors have no conflicts of interest to declare.

\section{Funding Sources}

This research did not receive any specific grant from funding agencies in the public, commercial, or not-for-profit sectors.

\section{Author Contributions}

Study conception and design: Erika Barbieri, Damiano Gentile, and Carlotta La Raja. Drafting of the manuscript: Erika Barbieri, Damiano Gentile, and Carlotta La Raja. Critical revision and final approval: all authors. 


\section{Case Reports in Oncology}

\begin{tabular}{l|l}
\hline Case Rep Oncol 2020;13:1158-1163 \\
\hline DOI: 10.1159/000510001 & $\begin{array}{l}\text { ○ 2020 The Author(s). Published by S. Karger AG, Basel } \\
\text { www.karger.com/cro }\end{array}$ \\
\hline
\end{tabular}

Barbieri et al.: Carcinoma of Unknown Primary Syndrome from Occult Breast Cancer

\section{References}

1 Krämer A, Hübner G, Schneeweiss A, Folprecht G, Neben K. Carcinoma of unknown primary - an orphan disease? Breast Care (Basel). 2008;3(3):164-70.

2 Stella GM, Senetta R, Cassenti A, Ronco M, Cassoni P. Cancers of unknown primary origin: current perspectives and future therapeutic strategies. J Transl Med. 2012 Jan;10:12.

3 Zaun G, Schuler M, Herrmann K, Tannapfel A. CUP syndrome - metastatic malignancy with unknown primary tumor. Dtsch Arztebl Int. 2018 Mar;115(10):157-62.

4 Halsted WS. I. The results of radical operations for the cure of carcinoma of the breast. Ann Surg. 1907 Jul; 46(1):1-19.

5 Walker GV, Smith GL, Perkins GH, Oh JL, Woodward W, Yu TK, et al. Population-based analysis of occult primary breast cancer with axillary lymph node metastasis. Cancer. 2010 Sep;116(17):4000-6.

6 Fizazi K, Greco FA, Pavlidis N, Daugaard G, Oien K, Pentheroudakis G. Cancers of unknown primary site: ESMO Clinical Practice Guidelines for diagnosis, treatment and follow-up. Ann Oncol. 2015 Sep;26(5):v133-8.

7 Ettinger DS, Agulnik M, Cates JM, Cristea M, Denlinger CS, Eaton KD, et al. NCCN Clinical Practice Guidelines Occult primary. J Natl Compr Canc Netw. 2011(Dec);9(12):1358-95.

8 Pentheroudakis G, Lazaridis G, Pavlidis N. Axillary nodal metastases from carcinoma of unknown primary (CUPAx): a systematic review of published evidence. Breast Cancer Res Treat. 2010 Jan;119(1):1-11.

9 Schorn C, Fischer U, Luftner-Nagel S, Westerhof JP, Grabbe E. MRI of the breast in patients with metastatic disease of unknown primary. Eur Radiol. 1999;9(3):470-3.

10 Kwee TC, Kwee RM. Combined FDG-PET/CT for the detection of unknown primary tumors: systematic review and meta-analysis. Eur Radiol. 2009 Mar;19(3):731-44.

11 Yang H, Li L, Zhang M, Zhang S, Xu S, Ma X. Application of neoadjuvant chemotherapy in occult breast cancer: five case reports. Medicine (Baltimore). 2017 Oct; 96(40):e8200.

12 Vlastos G, Jean ME, Mirza AN, Mirza NQ, Kuerer HM, Ames FC, et al. Feasibility of breast preservation in the treatment of occult primary carcinoma presenting with axillary metastases. Ann Surg Oncol. 2001 Jun;8(5): 425-31.

13 Merson M, Andreola S, Galimberti V, Bufalino R, Marchini S, Veronesi U. Breast carcinoma presenting as axillary metastases without evidence of a primary tumor. Cancer. 1992 Jul;70(2):504-8.

14 van Ooijen B, Bontenbal M, Henzen-Logmans SC, Koper PCM. Axillary nodal metastases from an occult primary consistent with breast carcinoma. Br J Surg. 1993 Oct;80(10):1299-300.

15 Khandelwal AK, Garguilo GA. Therapeutic options for occult breast cancer: a survey of the American Society of Breast Surgeons and review of the literature. Am J Surg. 2005 Oct;190(4):609-13. 